\title{
Scope of bacteriocins as a viable alternative to the traditional antibiotics
}

\begin{abstract}
Microbes produce an extradinary array of microbial defense systems. These include broad spectrum low molecular weight classical antibiotics, metabolic by-products, lytic agents, numerous types of protein exotoxins, defense bacteriophages and bacteriocins. Bacteriocins are defined as biologically active protein moieties with a bactericidal mode of action, which are ribosomally synthesized. They can alter cell membrane integrity and interfere with transcription, translocation or DNA replication. They differ from traditional antibiotics in one critical way that they have relatively narrow killing spectrum and are mostly toxic to bacteria, which are closely related strains. The intrinsic property of bacterial strains has led to studies for their potential use as bio preservatives, therapeutic agents and biocontrol organisms. In this review, some of the aspects of bacteiocinogeny particularly their prevalence in microbial world have been discussed.
\end{abstract}

Keywords: bacteriocins, bacillus, food preservative, antibiotics, alternate medicine
Volume 5 Issue 2 - 2016

\author{
Neelamegam Rameshkumar,' Govindarajan \\ RK, ${ }^{2}$ Muthukalingan Krishnan,' Nagarajan \\ Kayalvizhi \\ 'Department of Environmental Biotechnology, Bharathidasan \\ University, India \\ ${ }^{2}$ Department of Zoology, Periyar University, India
}

\begin{abstract}
Correspondence: Nagarajan Kayalvizhi, Assistant Professor, School of Lifes Sciences, Department of Zoology, Periyar University, Salem-636 01 I, Tamil Nadu, India, Tel 91+944-286507-2, Email kayalvizhinagarajan@gmail.com
\end{abstract}

Received: June 20,2016 | Published: November 22, 2016

\section{Introduction}

Microorganisms produce a remarkable array of antimicrobial substances which help them to compete in their limited environment for nutritional resources. These antimicrobial substances include broad-spectrum antibiotics, metabolic products (organic acids), lytic agents (lysozymes), various exotoxins and bacteriocins. Bacteriocins are biologically active protein moieties with a bactericidal mode of action. Over the past few decades, extensive use of antibiotics has led to the development of resistance in bacterial strains resistant to antibiotics. The infections lead prolonged hospital stays and antibiotic resistance are responsible for enormous economic and logistical burden on the health care systems. ${ }^{1}$ For example, in the United States, the loss due to antibiotic resistance is estimated to be \$US 3 billion per year, with an associated indirect loss of approximately \$US 30 billion. It is estimated that $74 \%$ of the gastrointestinal infections stems from the contaminated food, with $68 \%$ of these cases involving meat products. ${ }^{2}$ Canada's medical community reports over 5000-7000 confirmed cases of food poisoning annually. However, it is believed that 2.3million cases of food-borne disease actually occur every year in Canada. In turn, the financial toll of dealing with these infections has been estimated at approximately $\$$ CDN 3.7 billion. $^{2}$ As such, these properties make bacteriocins suitable and attractive for use as food bio-preservatives. ${ }^{3,4}$ Under this circumstance there is need for the use of newer antimicrobial agents in food preservation and discovery of novel antibiotics to address this health related issue.

As a consequence of rising incidence of antibiotic resistance in microorganisms, several research initiatives have begun to explore the application of naturally produced and genetically modified bacteriocins as alternative to the traditional antibiotics. The knowledge has been acquired during the past two decades and new groups of antimicrobial peptides have been discovered for the treatment of bacterial and fungal infections. Bacteriocins are often considered more natural in contrast to the currently used antibiotics, because they are thought to be present in many of the foods eaten since ancient times.
Bacteriocins are ribosomally synthesized antimicrobial peptides that are active against related species (narrow spectrum), or across genera (broad spectrum). They are also involved in the host defense and cell signaling mechanisms with bacteriocidal mode of action. Bacteriocin producing organisms are immune to their own bacteriocins and that are mediated by specific immunity proteins. They are produced by Gram-negative and Gram-positive bacteria and some members of the Archaea. There is also an ever-increasing interest in the use of antagonistic bacteria as biocontrol agents for the management of fungal and bacterial phytopathogens and, more recently, as probiotic agents in food formulations.

The use of antibiotics for the treatment of animals suffering from parasitic or bacterial infections is rarely questioned as long as the diseases have been diagnosed correctly and the dosage and duration of treatment follow prescriptive measures. But even for these therapeutic applications, it would be desirable to find compounds with fewer ties to human antimicrobials therapies. Reports on factors affecting the production of bacteriocins are limited, except for Nisin, a well-studied bacteriocin. ${ }^{5,6}$ Nisin and other bacteriocins produced by a variety of Gram positive and Gram negative bacteria have received a great deal of attention because they are considered to be beneficial for human health and food production. The nisin is the only bacteriocin has GRAS (Generally Recognized as Safe) status (21 CFR 184.1538) among all the bacteriocins types so far reported. The present review provides a general overview of bacteriocins, classification and potential applications in human and animal health as alternatives to conventional antibiotics.

\section{Classification of bacteriocins}

Bacteriocin family includes a diverse of proteins, varies in size, microbial target, mode of action, release, and immunity mechanisms. Bacteriocins are classified depending on their structures into three main groups. ${ }^{7,8}$ Class I bacteriocins are lantibiotics (lanthionine-containing antibiotics) in which unusual lanthionine and $\beta$-methylllanthionione residues are included during post-translational processing as covalent 
bridges between specific amino acids in their peptide chains. ${ }^{9}$ Currently, lantibiotics are divided into types A (relatively linear and flexible, cationic peptides), B (more globular, rigid peptides with no or negative net charge) and (two-component bacteriocins). ${ }^{9}$ They are active against most Gram-positive bacteria and often bind to lipid II, a precursor of peptidoglycan in the bacterial cell membrane.$^{10}$ Class I bacteriocins have a range of activities generally resulting in membrane destabilization, pore formation and/or inhibition of cell-wall synthesis through binding to specific lipids. ${ }^{9}$ Class II bacteriocins are large and highly diverse group of unmodified peptides with disulphide bridges are crucial for defining the 3-D conformations. They affect target cells in similar ways as class I bacteriocins, but their receptors seem to be proteins rather than lipids. ${ }^{11}$ Class III bacteriocins are not peptides but heat-labile proteins. Other classes of bacteriocins carry essential lipid or carbohydrate moieties and cyclic peptides. ${ }^{8}$

\section{Multidimensional features of bacteriocins and its applications}

Bacteriocins are attractive candidates for the use in food and agricultural industries. For example, bacteriocin-producers are used in animal health feed for pigs and other livestock and they protect animals from severe gastrointestinal infections. ${ }^{12}$ Lantibiotics such as nisin and lacticin are widely used as preservative agents in meat and dairy products. ${ }^{9}$ Bacteriocins have also been used in human health and medical applications. The lantibiotic lacticin 3147 displays potent activity against virulent Multidrug Resistant Staphylococcus aureus (MRSA) and Vancomycin Resistant Enterococci (VRE) bacterial strains. ${ }^{13}$ Effectiveness against clinically important pathogens highlights the potential use of these bacteriocins for therapeutic applications against bacterial infections.

In particular, bacteriocins have several desirable properties that make them suitable for food preservation. They

i. are generally recognized as safe substances,

ii. are not active and nontoxic on eukaryotic cells,

iii. inactivated by digestive proteases, hence little influence on the gut microbiota,

iv. are usually $\mathrm{pH}$ and heat-tolerant,

v. they exhibit broad antimicrobial spectrum, against many foodborne pathogens and spoilage bacteria,

vi. they show a bactericidal mode of action, usually acting on the cytoplasmic membrane and therefore no cross resistance to antibiotics, and

vii. their genetic determinants are usually plasmid-encoded. More recently Kayalvizhi et al. ${ }^{14}$ reported the stable nature of the bacteriocin to high temperature and resistant to various chemicals it also exhibited antimicrobial activity against food-borne pathogens make this bacteriocin as potent attractive antimicrobial agent in food products.

The empirical use of microorganisms and/or their natural products for the preservation of foods (biopreservation) has been a common practice in the history of mankind. Bacteria from LAB have attracted great interest in terms of food safety and the only bacteriocin approved for utilization as a preservative in many foods by the
US Food and Drug Administration is nisin, which is commercially available as Nisaplin ${ }^{\mathrm{TM}}$ (Danisco, Copenhagen, Denmark). Another most studied bacteriocin-producing bacteria are B. subtilis and $B$. licheniformis, which are "generally recognized as safe" (GRAS) bacteria. ${ }^{15}$ The genus Bacillus includes a variety of industrially important bacterial species and has a history of safe use in both food and industry. ${ }^{16}$ The potential of Bacillus to produce antibiotics and peptides has been recognized for more than 50 years. Recombinant $B$. licheniformis strains have been designed and used extensively in large scale fermentations. ${ }^{17}$ Bacillus spp., have been extensively used in industrial production of amylases, proteases, antibiotics, insecticides and special chemicals with low risk of adverse effects to human health or the environment. ${ }^{18,19}$ Interestingly, a novel broadspectrum bacteriocin was purified and characterized from Bacillus licheniformis MKU3, which was able to inhibit the growth of several pathogenic strains..$^{20}$ In addition, Kayalvizhi et al. ${ }^{21}$ has reported the applicability of bacteriocin which could be used as a biopreservation of animal skins by getting rid of chemical preservatives. Bacteriocins such as nisin and lacticin are widely used as preservative agents in meat and dairy products. Recently, the importance of bacteriocins in human health and medical applications has been demonstrated. The lacticin 3147 displays potent activity against virulent bacterial strains such as methicillin resistant Staphylococcus aureus (MRSA) and vancomycin resistant Enterococci (VRE). Recently, Kayalvizhi et al. ${ }^{14}$ identified and cloned a new bacteriocin from Bacillus licheniformis which showed a broad spectrum of activity against Gram-positive and Gram-negative bacteria. All together effectiveness of bacteriocins against clinically important pathogens highlights their immense potential in therapeutic applications.

\section{Conclusion}

This is a promising field of research, because many bacteriocins from bacilli may solve the limitations of LAB bacteriocins due to their sometimes broader inhibitory spectra against Gram negative bacteria and even against fungi. Hopefully, the number of patents on applications of Bacillus bacteriocins will increase in the near future, and the antimicrobial arsenal of this group of bacteria, which are widely disseminated and adapted to survive under extreme conditions, will be exploited in a more rational way. Last, but not the least, bacteriocinsproducing strains could be exploited for environmental applications such as, for example, in the control of SRB causing bio corrosion. The bacteriocins production capacity could be exploited as a selective advantage for the proliferation of strains with technological interest in natural or even industrial environments. Furthermore, genomic and proteomic approaches in the field of bacteriocins could improve our understanding to fully exploit bacteriocins as a potential alternative to antibiotics or development of novel drug for the mankind.

\section{Acknowledgements}

Dr. N Kayalvizhi gratefully acknowledges the Department of Science and Technology for the award of DST WOS-A, New Delhi, India for the financial assistance through Women Scientist Scheme (SR/WOS-A/LS-629/2012).

\section{Conflict of interest}

The author declares no conflict of interest. 


\section{References}

1. Evans HL, Lefrack SN, Lyman J, et al. Cost of gram negative resistance. Crit Care Med. 2007;35(1):89-95.

2. Henson SJ, Majowicz SE, Masakure O, et al. Estimating the economic cost of acute gastrointestinal infection in Canada: results from the national studies on acute gastroinstentinal illness in British Columbia. International Food Economy Research group. Canada: Univesity of Guelph; 2007.

3. De la Fuente Salcido NM, Barboza-Corona JE. Harmlessness and food preservation. Acta Universitaria. 2010;20:43-52.

4. Udhayashree N, Senbagam D, Senthilkumar B, et al. Production of bacteriocin and their application in food products. Asian Pacific Journal of Tropical Biomedicine. 2012;2(1):S406-S410.

5. Penna TC, Moraes DA. Optimization of nisin production by Lactococcus lactis. Applied Biochemistry and Biotechnology. 2002;98(1):775-789.

6. Kayalvizhi N, Gunasekaran P. Production and characterization of a low-molecular-weight bacteriocin from Bacillus licheniformis MKU3. Lett Appl Microbiol. 2008;47(6):600-607.

7. Cotter PD, Hill C, Ross RP. Bacteriocins:developing innate immunity for food. Nat Rev Microbiol. 2005;3(10):777-788.

8. De Jong A, van Hijum SAFT, Bijlsma JJE, et al. BAGEL:a web-based bacteriocin genome mining tool. Nucleic Acids Res. 2006;34:W273279.

9. Chatterjee C, Paul M, Xie L, et al. Biosynthesis and mode of action of lantibiotics. Chemical Reviews. 2005;105(2):633-684.

10. Wiedemann I, Bottiger T, Bonelli RR, et al. The mode of action of the lantibiotic lacticin 3147-a complex mechanism involving specific interaction of two peptides and the cell wall precursor lipid II. $\mathrm{Mol}$ Microbiol. 2006;61(2):285-296.

11. Diep DB, Skaugen M, Salehian Z, et al. Common mechanisms of target cell recognition and immunity for class II bacteriocins Proceedings of National Academy of Sciences. 2007;104(7):2384 2389.
12. Kirkup BC. Bacteriocins as oral and gastrointestinal antibiotics:theoretical considerations, applied research, and practical applications. Curr Med Chem. 2006;13(27):3335-3350.

13. Martin NI, Sprules T, Carpenter MR, et al. Structural characterization of lacticin 3147, a two-peptide lantibiotic with synergistic activity. Biochemistry. 2004;43(11):3049-3056.

14. Kayalvizhi N, Rameshkumar N, Gunasekaran P. Cloning and characterization of mersacidin like bacteriocin from Bacillus licheniformis MKU3 in Escherichia coli. Journal of Food Science Technology. 2016;53(5):1-9.

15. Schallmey M, Singh A, Ward OP. Developments in the use of Bacillus species for industrial production. Can J Microbiol. 2004;50(1):1-17.

16. Paik SH, ChakicherlaA, Hansen JN. Identification and characterization of structural and transporter genes for, and the chemical and biological properties of sublancin 168, a novel lantibiotic produced by Bacillus subtilis 168. J Bio Chem. 1998;273(36):23134-23142.

17. Outtrup H, Jorgensen ST. The importance of Bacillus species in the production of industrial enzymes. In: Applications and Systematics of Bacillus and Relatives. Berkeley RCW, et al. editors. Oxford: Blackwell; 2002.

18. Mendo S, Faustino NA, Sarmento A, et al. Purification and characterization of a new peptide antibiotic produced by a thermotolerant Bacillus licheniformis strain. Biotechnology Letter. 2004;26(2):115-119.

19. Korenblum E, Von Der Weid I, Santos ALS, et al. Production of antimicrobial substances by Bacillus subtilis LFE-1, B. firmus $\mathrm{H} 2 \mathrm{O}-$ 1 and B. licheniformis T6-5 isolated from an oil reservoir in Brazil. J Appl Microbiol. 2005;98(3):667-675.

20. Kayalvizhi N, Gunasekaran P. Purification and characterization of a novel broad-spectrum bacteriocin from Bacillus licheniformis MKU3. Biotechnology and Bioprocess Engineering. 2010;15(2):365370 .

21. Kayalvizhi N, Anthony T, Sathyavathi S, et al. Biodegradation and Biopreservation of animal skins. 44th Leather Research Industry Get-Together (LERIG); 2010. 